\title{
Identification and Validation of the Lactate Threshold Velocity using the Y-Intercept and Slope of the Fit Curve (YIS Method)
}

\author{
Emma Swanwick $^{1}$, David B. Pyne ${ }^{2}$, Bernard Savage ${ }^{3}$ and Martyn Matthews ${ }^{1 *}$ \\ ${ }^{1}$ School of Health Sciences, University of Salford, UK \\ ${ }^{2}$ Department of Physiology, Australian Institute of Sport, Australia \\ ${ }^{3}$ GADI Research Centre, University of Canberra, Australia
}

*Corresponding author: Martyn Matthews, School of Health Sciences, University of Salford, Frederick Road, Salford M6 6PU, UK, E-mail:m.j.matthews@salford.ac.uk

\begin{abstract}
Purpose: To present a new method for determining the individual Lactate Threshold velocity (LTV) in trained swimmers using the intercept and slope (YIS) of the Lactate-Velocity curve (La-V).

Methods: Forty-two national-level swimmers undertook a $7 \times 200 \mathrm{~m}$ discontinuous incremental swimming step test. The LTV was determined by a mathematical formula that calculated the Lactate Threshold (LTV) as a function of the $y$-intercept and slope of the Lactate-Velocity curve (YIS method, where LTv $=-0.217 \times$ [y-intercept $(\mathrm{La}-\mathrm{V}) /$ slope $(\mathrm{La}-$ $\mathrm{V})$ ] and compared to an existing Fixed Slope (FSM) Method, which had been seen to be the most consistent of 4 methods and used a similar slope protocol. Subsequently, on three occasions over a six-week period, using twelve different swimmers, the method of determining LTV was compared to three practical methods of validating the LTv: a $2000 \mathrm{~m}$ time trial, $24 \times 100 \mathrm{~m}$, and $10 \times 300 \mathrm{~m}$ swim sets.

Results: The slope of the La-V curve for Freestyle was significantly different between YIS and FSM $(p=0.03)$. The mean lactate at LT with the YIS method $(3.2 \pm 0.4 \mathrm{mM})$ was significantly lower than FSM $(3.4 \pm 0.3 \mathrm{mM})$. Swim velocity at LT was not significantly different between $\mathrm{YIS}$ and the $\mathrm{FSM}, \mathrm{p}=0.40$.

Conclusions: The YIS method for determining the LT and LTv, derived from the $7 \times 200 \mathrm{~m}$ incremental step test, provides an objective means of quantifying the lactate threshold in swimmers.
\end{abstract}

\section{Keywords}

Swimming, Blood lactate, Anaerobic threshold, Step test, Lactate-velocity curve, Slope

\section{Introduction}

Although the Lactate Threshold (LT) has lost some of its status as a marker of exercise intensity, the LT does mark a metabolic transition that, when we are looking to explain energy timelines, can provide an important perspective. This paper is the first of a series looking to clarify and transform how we monitor intensity of exercise. As such we need to create a more robust method of identifying LT.

The LT has been typically defined as the highest constant workload that can be sustained without incurring progressive increases in blood lactate concentration [1-3]. A number of methods have been used to detect the exact exercise intensity at which it occurs. Initial attempts with visual detection were open to considerable experimental error [4]. Lactic acid concentration in the blood may follow an exponential rise rather than a 'threshold break' in its dynamics, which further underlines the necessity of a valid, reliable and accurate means of identifying the LT. The lactate profile is further influenced by the specific sport and event in which the athlete trains and the length of time spent on specific training tasks [5].

A number of graded and progressive exercise test protocols have evolved for the determination of the Lactate Threshold velocity (LTV). Initially a standard reference point such as the $4 \mathrm{mM}$ was used to identify the velocity at submaximal workloads $[2,6,7]$. This appears 
to be appropriate for athletes in endurance-based activities wand where the repeats used are longer than 4 minutes (or where a steady state may be reached). In many sports, such as in swimming, the events last less than 8 minutes (typically 45 secs to 3 minutes). Adopting a fixed $4 \mathrm{mM}$ may lead to over-estimation of workloads in training as it has been found that the workload and duration of the graded exercise tests influences the determination of LTV [8-11]. Careful selection of protocol is therefore important if meaningful results are to be obtained, with alterations of work-load level and duration changing the corresponding threshold value in graded exercise tests [3]. This demonstrates that the level of lactate in the blood is dependent upon workload intensity and duration [3]. Discontinuous graded exercise tests allow some lactate elimination, leading to a shift of the lactate curve toward the higher speeds for a set intensity [12].

A number of investigators have proposed methods to estimate both LT and LTV by mathematical techniques [13-15]. The blood lactate concentration of the calculated LT between the different mathematical models can differ by $2 \mathrm{mM}$ from the same data [6]. Borch, et al. [1] suggested the use of an exponential function $\mathrm{La}=$ $\mathrm{a}^{*} \mathrm{edx}+\mathrm{c}$ and identified the point on this curve that related to a fixed slope of 0.086 [1]. It was reported in this paper that this point was related favorably to a lactate level of $3.0 \mathrm{mM}$. The possibility of over-estimating LTV is increased with a fixed slope method as it ignores individual variation elicited by genetic pre-determinants, training background, and specificity of sporting activities.

There is no universally accepted method that calculates a valid estimate of LT as a function of the lactate-velocity profile using a discontinuous, incremental test (such as the $7 \times 200 \mathrm{~m}$ swimming step test) using shorter repeat periods (2-3 minutes). The aim of this study was to describe a specific method for calculating the point of LT. The proposed Y-Intercept Slope (YIS) method was compared with the Fixed Slope (FSM) Method. Other methods (D-Max and Fixed Tangent) had already been found to be less reliable. The ability of the YIS method to assess changes longitudinally over a six-week period in the same stroke (freestyle) was also investigated.

\section{Methods}

\section{Subjects}

Forty-two international standard swimmers from the Australian Institute of Sport and New Zealand national squad. All subjects were male and aged between 17 and 24 years. Freestyle was the stroke used for all tests. All subjects provided written informed consent for the physiological testing procedures, which had prior approval from the Ethics Committee of the Australian Institute of Sport.

\section{Study design}

The subjects performed a graded sub-maximal swimming test of $7 \times 200$-meter freestyle swims as a basis for the assessment of aerobic fitness. This testing was conducted in accordance with the protocols of the Australian National Swimming Team [16]. For all subjects, training consisted of a mixture of low, moderate and maximal intensity aerobic work, short sprints and poolbased stroke specific drills and exercises. Weekly swimming training volume averaged $54 \pm 19 \mathrm{~km}$. week $\mathrm{k}^{-1}$ (mean \pm SD) over the six weeks. All swimmers also carried out land-based training between three and five times per week consisting of resistance training circuits, strength training and flexibility work.

\section{Lactate profiling}

$7 \times 200 \mathrm{~m}$ step test was conducted on a 5:30 $\mathrm{min}$ cycle. Dietary intake, 24-hour prior training load, and pre-test warm-up were all standardized for each test. The seven swims were performed at an even pace, controlled by the swimmer. Each swimmer was provided with individual target times based upon the personal best performance on freestyle over $200 \mathrm{~m}$. The range of target times was approximately 30 seconds and progressed from slowest to fastest in consistent increments $[16,17]$. This progression equates to an approximate rise in intensity from $70 \%$ of maximal (first swim) to $100 \%$ of maximal effort (seventh swim).

Each $200 \mathrm{~m}$ swim began with a push start while partially submerged in the water. Mean swimming velocity over the $200 \mathrm{~m}$ was recorded for each effort as well as the $100 \mathrm{~m}$ split times using a manual stopwatch. Stroke count was recorded for each 50-meter lap to allow the calculation of the stroke characteristics (distance per stroke and the stroke rate). Heart rate was monitored immediately on completion of each swim with a Polar heart rate monitor (Polar Electro OY, Kempele, Finland). Capillary blood $(25 \mu \mathrm{l})$ was collected from finger-tip or ear lobe puncture one minute after the completion of each swim. Lactate concentration was analyzed by a Lactate Pro Lactate meter (Axon Labs., Austria). All tests were completed at the same time of day on the three different occasions for each swimmer.

\section{Validation study}

As a means of validating the method, an additional twelve swimmers from the South Australian Swimming Distance Squad were required to undergo the $7 \times 200$ $\mathrm{m}$ swimming step test plus three additional tests in the pool over a 9-day period (see Figure 1 ). On day 0 all subjects undertook the $7 \times 200$ swimming step test described above. On day 3 all subjects undertook simultaneously an interval set of $10 \times 300 \mathrm{~m}$. On day 6 the subjects undertook a second standardized interval set of $24 \times 100 \mathrm{~m}$ intervals. Finally, on day 9 the swimmers completed a continuous maximal effort $2000 \mathrm{~m}$ time trial (2000TT). This schedule of testing was designed to 


\begin{tabular}{|c|c|c|c|}
\hline $\begin{array}{l}7 \times 200 \mathrm{~m} \\
\text { Step Test }\end{array}$ & $2000 \mathrm{~m}$ & $24 \times 100 \mathrm{~m}$ & $10 \times 300 \mathrm{~m}$ \\
\hline \begin{tabular}{l|l} 
& \\
\end{tabular} & + & + & + \\
\hline 1 & 3 & 6 & 9 \\
\hline
\end{tabular}

Figure 1: Schedule of swimming tests for calculation of LTV using different methods.

validate the YIS methods in three separate ways: i) Comparison with the existing FSM mathematical technique; ii) Comparison with two standard interval training sessions; iii) Comparison with a continuous 2000TT.

\section{Validating the YIS method}

Determination of YIS and FSM Lactate Thresholds The first means of validating the YIS method compared the calculated LTV value with that obtained with the previously described FSM method. The Y-Intercept Slope method (YIS) calculates the LTV from the slope and $y$-intercept of the blood lactate - velocity curve, as distinct from the point of onset of blood lactate accumulation. This method is based upon determining the $y$-intercept (blood lactate concentration), which is the regression line through the data points ( $x$ 's and $y$ 's in this case for swimming velocity and lactate concentration), returning the point (lactate concentration at LT) where the regression line intersects the $y$-axis of the paired data. The LTV ( $x$-value) is calculated from the LT (mM) ( $y$-value) by inserting this value into the regression equation (see Figure 1). Initial pilot work showed that YIS analysis using the abbreviated upper portion of the lactate-velocity curve from the $4^{\text {th }}$ to the $7^{\text {th }}$ data points (as opposed to the full lactate curve using all 7 data points) generated a value that correlated more closely with other estimates of the LTV. The modified FSM method involves calculating the point that yields the maximal perpendicular distance from the lactate curve to a tangent line formed from the aerobic threshold (at the velocity corresponding with $0.4 \mathrm{mM}$ rise in lactate concentration over the baseline) to the peak lactate value [18].

Interval Training Sessions - The second means of validating the calculated LTV using the YIS method was a comparison against the mean velocity achieved in two standard swimming interval training sessions. The first session consisted of $24 \times 100 \mathrm{~m}$ intervals on a 1:30 (min:sec) cycle. The second session consisted of $10 \times 300$ $\mathrm{m}$ intervals on 4:30 (min:sec) cycle. The swimmers were asked to swim each interval at an effort corresponding to a heart rate of approximately $160 \pm 5$ beats per min. These sessions are typical of those used in competitive swimming as anaerobic or lactate threshold training sessions. Heart rate and lactate was measured at the completion of each 100 or $300 \mathrm{~m}$ effort using the same protocols as stated above except lactate was only taken at the completion of each set. The time for each effort was recorded manually with a Seiko S101A stop watch (Seiko Corporation, Japan).

$2000 \mathrm{~m}$ Time Trial - A third means of validating the YIS method was a comparison against the mean velocity achieved in a continuous maximal effort $2000 \mathrm{~m}$ time trial. For this test the athletes were instructed to swim the required $2000 \mathrm{~m}$ as fast and as evenly as possible. Total time for the $2000 \mathrm{~m}$ and $100 \mathrm{~m}$ split times were recorded using a Seiko S101A stop watch (Seiko Corporation, Japan). The $1^{\text {st }}$ and last $100 \mathrm{~m}$ split were dropped for the calculation of average $100 \mathrm{~m}$ time throughout the swim. All athletes performed the freestyle stroke. Heart rate and blood lactate concentration was measured within 60 secs of completion of the time trial with as with the previously stated protocols.

\section{Calculation of YIS for determining LT}

The Y-Intercept Slope method (YIS) calculates the LT as distinct from the point of Onset of Blood Lactate Accumulation (OBLA). The YIS method is based upon determining the intercept which is the regression line through the data points ( $x$ 's and y's in this case of swimming velocity and lactate concentration), returning the point where the regression line intersects the $y$ axis of paired data where:

The intercept $=$

(1) $a=y-b x$

and the slope $b=$

$(2) n x y-(\Sigma x)(\Sigma y)$

$\mathrm{n} \Sigma \mathrm{x}^{2}-\left(\sum \mathrm{x}\right)^{2}$

The slope is the vertical distance divided by the horizontal distance between two points of the profile. This value indicates the rate of change between points on this profile. By dividing the intercept by the slope of the profile and multiplying it by a constant the lactate value at the LT is estimated.

$$
\mathrm{LT}=\text { (3) }-0.0217 \mathrm{xy}-\mathrm{bx}
$$

The methods of Borch, et al. [1] has been proposed as a means for identifying the LT. For comparison purposes, it was necessary to adopt a single criterion for identifying the difference between the calculated lactate values of the four methods. An example of this standardization was to determine the slope of each profile by the methods of Borch [1] and convert this to a tangent angle. This approach permitted a comparison of these two existing methods to the proposed YIS method for determining LT. The D-max method involves constructing a tangent from the lactate threshold to the peak lactate value [17].

\section{Statistical analysis}

The results are expressed as mean and standard deviation $(X \pm S D)$. Each profile of lactate and velocity (the La-V profile) was plotted and assessed for goodness of fit (Pearson's Product Moment correlation) with a $3^{\text {rd }}$ or- 


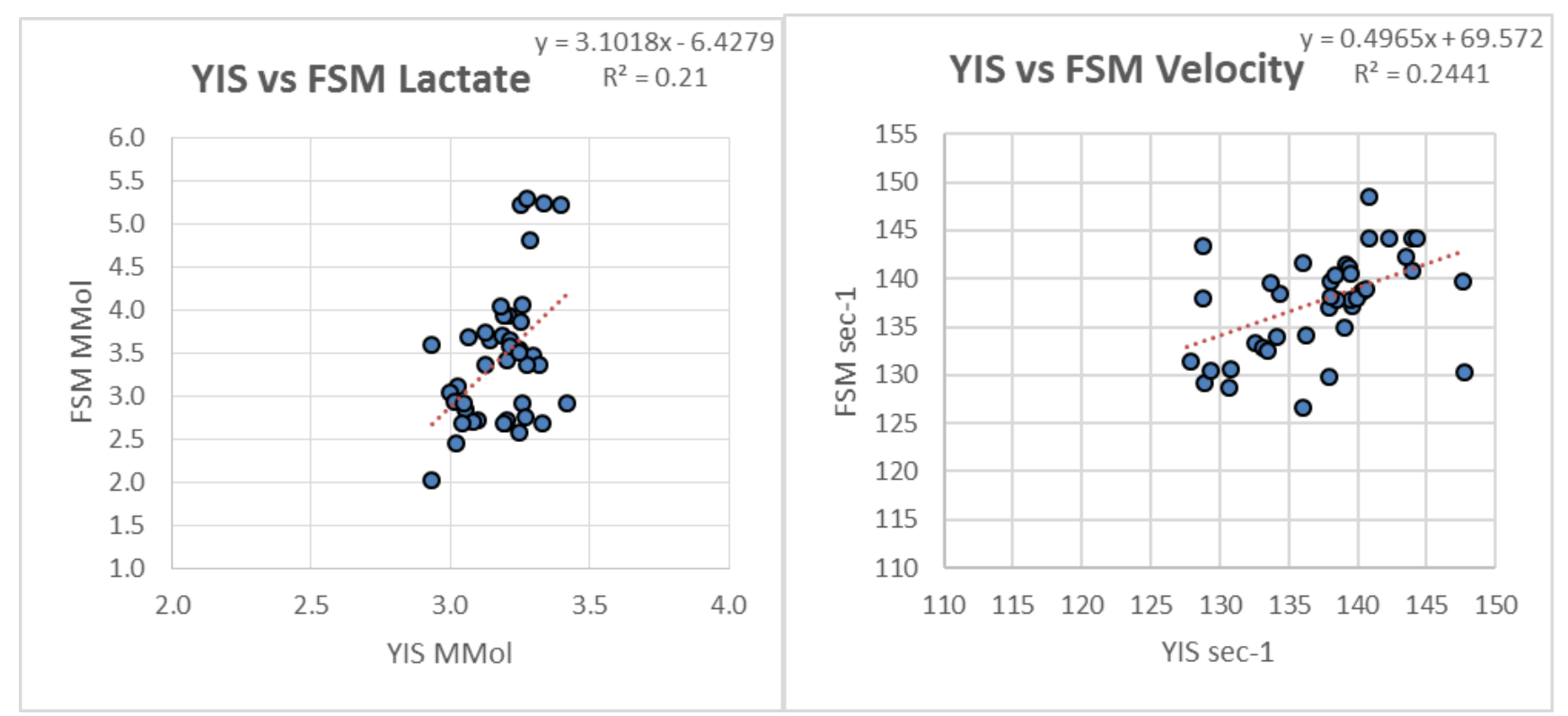

Figure 2: Relationship between YIS and FSM Lactate and YIS and FSM velocity.

Table 1: Mean profile data and for freestyle $(n=42)$.

\begin{tabular}{|l|l|l|l|}
\hline Mean & SD & Hi & \multicolumn{1}{l|}{ low } \\
\hline & \multicolumn{3}{l|}{ Time (Secs) } \\
\hline 146.3 & 5.8 & 157.4 & 133.8 \\
\hline 141.8 & 5.3 & 152.6 & 128.3 \\
\hline 137.4 & 4.7 & 148.0 & 126.2 \\
\hline 132.9 & 4.8 & 144.9 & 123.0 \\
\hline 128.8 & 4.3 & 141.8 & 122.4 \\
\hline 126.0 & 4.3 & 138.4 & 118.5 \\
\hline 123.6 & 4.8 & 135.1 & 114.8 \\
\hline & & Lactate (Mmol) \\
\hline Mean & SD & Hi & low \\
\hline 1.3 & 0.5 & 2.9 & 0.7 \\
\hline 1.6 & 0.6 & 2.8 & 0.6 \\
\hline 1.9 & 0.7 & 3.1 & 0.8 \\
\hline 2.6 & 1.0 & 4.4 & 0.8 \\
\hline 3.7 & 1.4 & 6.8 & 1.3 \\
\hline 5.6 & 2.1 & 9.3 & 2.0 \\
\hline 7.9 & 2.5 & 12.6 & 2.2 \\
\hline 11.4 & 2.5 & 16.2 & 6.3 \\
\hline & & Heart Rate (BPM) \\
\hline Mean & SD & Hi & low \\
\hline 136 & 15 & 160 & 90 \\
\hline 143 & 11 & 165 & 120 \\
\hline 152 & 11 & 170 & 130 \\
\hline 161 & 9 & 177 & 140 \\
\hline 169 & 9 & 186 & 147 \\
\hline 176 & 9 & 198 & 150 \\
\hline 183 & 11 & 210 & 160 \\
\hline & & & \\
\hline & & & \\
\hline
\end{tabular}

der polynomial plot. Student's t-test was carried out to determine statistical differences. Statistical analysis was completed with SPSS v19. Results were considered as significant when $P \leq 0.05$.

\section{Results}

\section{$7 \times 200$ m Step Test Results}

The results for the $7 \times 200 \mathrm{~m}$ step test are presented in Table 1. From the slowest to fastest efforts, freestyle times for the $200 \mathrm{~m}$ ranged from $146.3 \pm 5.8$ to $123.6 \pm$ $4.8 \mathrm{sec}$. These times are indicative of the highly-trained nature of the subject group. Blood lactate values ranged from $1.3 \pm 0.5$ to $11.4 \pm 2.5 \mathrm{mM}$. The calculated LT 200 $m$ times by the YIS method was: $137.2 \pm 5.1 \mathrm{sec}(86 \%$ of max swim pace).

\section{Comparison of predicted lactate concentration (mM) at LT}

The predicted lactate for swimming freestyle at the LT point, by use of the YIS method was $3.2 \pm 0.1 \mathrm{mM}$. This was lower than the turn point estimated by using the FSM method (mean $3.4 \pm 1.0 \mathrm{mM}, \mathrm{p} 0.01$ ). The Rsq value is shown in Figure 2. This figure demonstrates that although the LT detected by the different protocols appeared to be close, the relationship between them is poor (RSQ 0.2). There was a $91 \%$ likelihood of a mechanistic difference between the two methods. The change in mean was $0.3 \mathrm{mM}$ with confidence limits of 0.2 and 0.5 Table 2.

\section{Comparison of predicted AT pace}

The calculated swim pace at LT (seconds per 200 $\mathrm{m})$ determined by the two different methods was not significantly different between the predicting $200 \mathrm{~m}$ LT times of YIS $(137.2 \pm 5.1 \mathrm{sec})$, and the FSM method $(136.4 \pm 4.9 \mathrm{sec} ; p=0.3)$. The Rsq is shown in Figure 2. Again, although the means appear to be similar, the reality is that there is little agreement in the speed determined by the two methods in paired samples.

\section{Results of validation study}

The comparison data for the 5 methods of evaluation of LT and LTV are shown in Figure 2. The calculated LTV, mean heart rate, and mean blood lactate concentration using the YIS methods and the mean velocities obtained from the FSM, 2000TT, $24 \times 100 \mathrm{~m}$ and $10 \times$ 
Table 2: Comparison of the Lactate Threshold velocity (LTV), heart rate and blood lactate concentration at the LTV, for the YIS method compared with the $D_{\max }$ method, the 2000 TT, and the $24 \times 100 \mathrm{~m}$ and $10 \times 300 \mathrm{~m}$ training sets.

\begin{tabular}{|c|c|c|c|c|c|c|}
\hline Method & & YIS & FSM & 2000 TT & $24 \times 100 \mathrm{~m}$ & $10 \times 300 \mathrm{~m}$ \\
\hline \multirow{4}{*}{$\begin{array}{l}\text { Lactate Threshold } \\
\text { Velocity (sec/100 } \\
\text { m) }\end{array}$} & Mean & 76.2 & 75.1 & 76.8 & 76.3 & 77.4 \\
\hline & Std. Dev. & 3.7 & 3.8 & 3.5 & 3.5 & 3.8 \\
\hline & $95 \% \mathrm{CL}$ & 73.8-78.6 & $72.4-77.5$ & 74.6-79.1 & $74.1-78.5$ & $75.0-79.8$ \\
\hline & Post Hoc p-value & - & $p=0.00$ & $p=0.22$ & $p=0.99$ & $p=0.00$ \\
\hline \multirow{4}{*}{ Heart Rate (bpm) } & Mean & 167 & 164 & 178 & 168 & 174 \\
\hline & Std. Dev. & 9 & 10 & 8 & 9 & 7 \\
\hline & $95 \% \mathrm{CL}$ & $161-172$ & $158-170$ & $172-183$ & $162-173$ & $170-178$ \\
\hline & Post Hoc p-value & - & $p=0.74$ & $p=0.00$ & $p=0.98$ & $p=0.01$ \\
\hline \multirow{4}{*}{ Lactate (mM) } & Mean & 3.2 & 3.4 & 3.1 & 2.8 & 3.5 \\
\hline & Std. Dev. & 0.12 & 0.8 & 0.6 & 0.5 & 0.6 \\
\hline & 95\% C.I. & $3.1-3.3$ & $2.6-4.1$ & $2.7-3.4$ & $2.5-3.2$ & $3.1-3.9$ \\
\hline & Post Hoc p-value & - & $p=0.89$ & $p=0.42$ & $p=0.99$ & $p=0.01$ \\
\hline
\end{tabular}
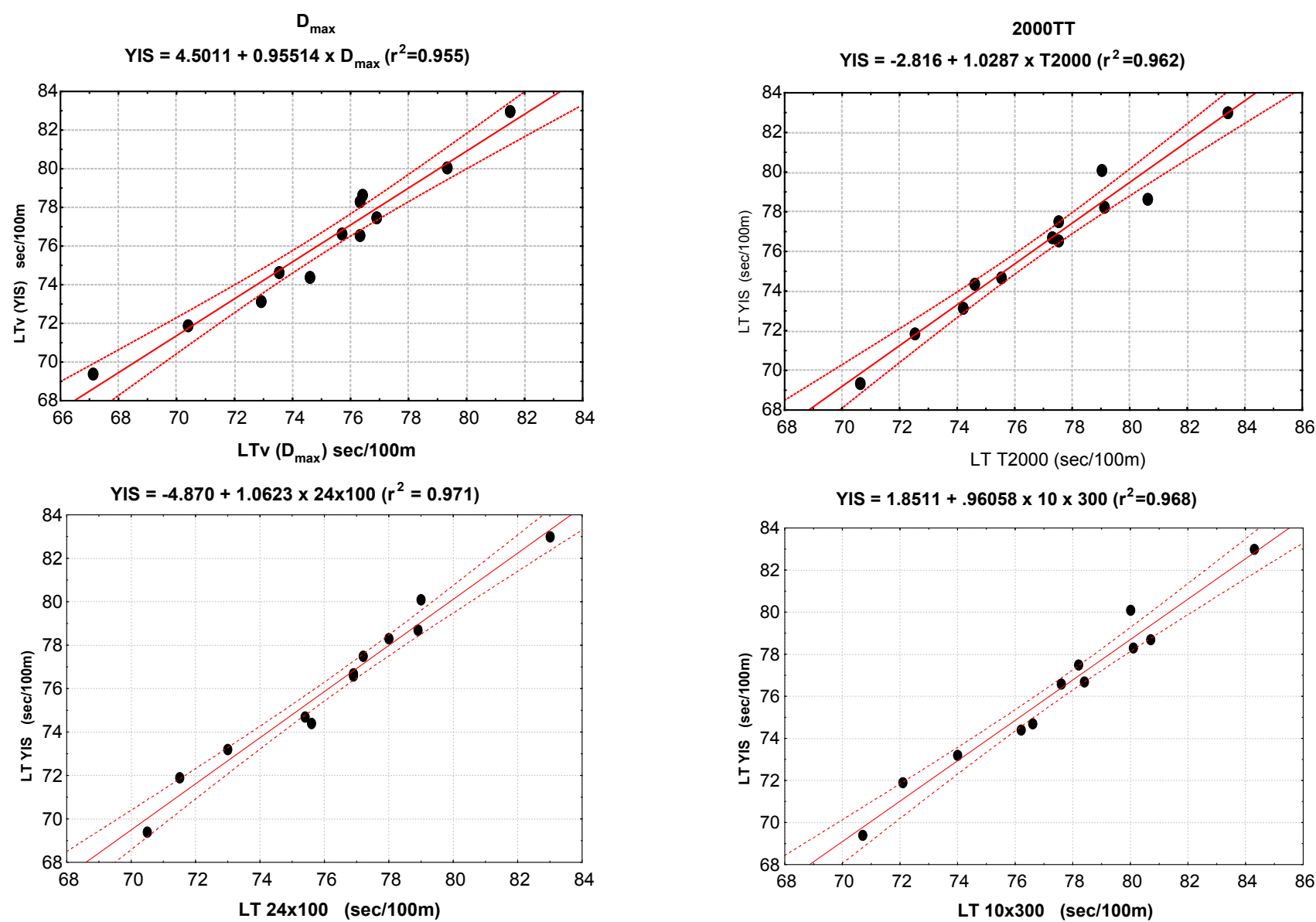

Figure 3: Correlation plots for calculated Lactate Threshold velocities (LTV) from a) YIS method from the $7 \times 200 \mathrm{~m}$ step test, b) 2000 TT, c) $24 \times 100 \mathrm{~m}$ interval training session, and d) $10 \times 300 \mathrm{~m}$ interval training session. Dashed lines indicated $95 \%$ Confidence Limits for the regression line.

$300 \mathrm{~m}$ training sets are shown in Table 2. The Mean LTV derived with the YIS method $(76.2 \pm 3.7 \mathrm{sec} / 100 \mathrm{~m})$ was similar to both the 2000TT (76.8 $\pm 3.6 ; p=0.22)$ and the $24 \times 100 \mathrm{~m}$ set $(76.3 \pm 3.5 ; p=0.99)$, but significantly slower than the FSM method $(75.1 \pm 3.7 ; p=0.00)$ and faster than the $10 \times 300 \mathrm{~m}$ set $(77.4 \pm 3.8 ; p=0.00)$ as shown in Table 2.

The mean blood lactate concentration at the LT with the YIS method $(2.7 \pm 0.4 \mathrm{mM})$ was similar to that of the 2000TT ( $3.1 \pm 0.6 ; p=0.42)$ and the $24 \times 100 \mathrm{~m}$ set $(2.8$ $\pm 0.5 ; p=0.99)$, but significantly lower than the $10 \times 300$ $m$ set: $3.5 \pm 0.6 ; p=0.11)$. No differences were observed for heart rate, at LTV for YIS (167 $\pm 9 \mathrm{bpm})$ and the $24 \times$ $100 \mathrm{~m}$ set (168 $\pm 9 \mathrm{bpm} ; \mathrm{p}=0.98)$, but YIS heart rate was significantly lower than the 2000TT $(178 \pm 8 \mathrm{bpm} ; \mathrm{p}=$ $0.00)$ and the $10 \times 300 \mathrm{~m}$ set $(174 \pm 7 ; p=0.01)$.

Correlation plots for calculated LTV using the YIS method compared with the FSM method, and the mean swimming velocities obtained from the $2000 \mathrm{TT}, 24 \times 100 \mathrm{~m}$, and $10 \times 300 \mathrm{~m}$ training sets are shown in Figure 3. All four comparisons show a high correlation $r>0.95$. The slope of the regression plots indicates the degree of agree- 

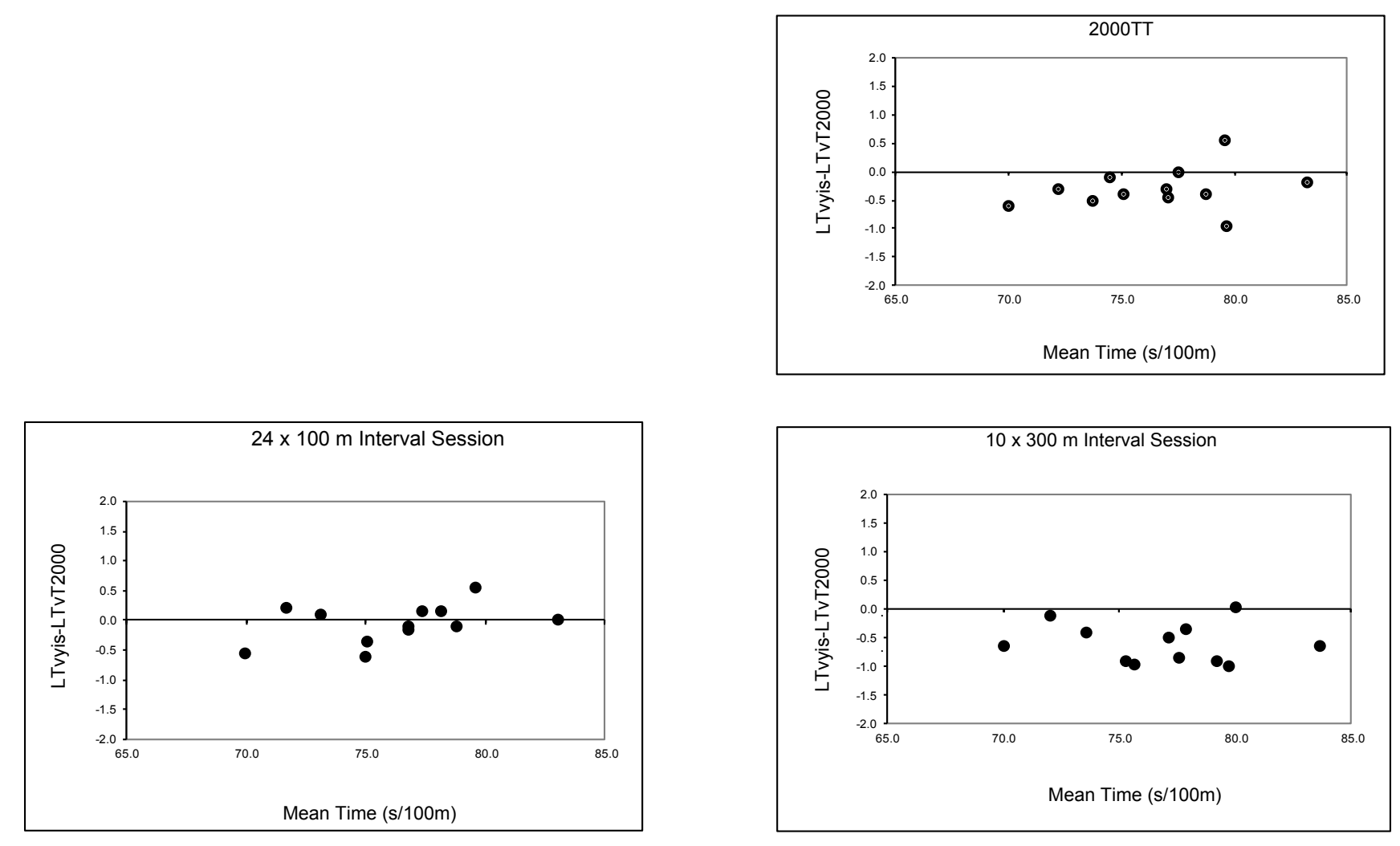

Figure 4: Bland-Altman level of agreement plots showing the relationship between LTV derived with the YIS method compared with: a) $2000 \mathrm{TT}$, b) $24 \times 100 \mathrm{~m}$ interval session, and c) $10 \times 300$ training session.

ment between the LTV detection methods and standard swimming training sets. The values in comparison with the YIS method were a factor of $0.96,1.03,1.06$, and 0.96 for the FSM, 2000TT, $24 \times 100 \mathrm{~m}$ and $10 \times 300 \mathrm{~m}$ sets respectively.

The level of agreement results are shown in Figure 4. These results indicate that the LTV values calculated with the YIS method are in good agreement with the mean velocities obtained from the criterion $2000 T T$ test, $24 \times 100 \mathrm{~m}$, and $10 \times 300 \mathrm{~m}$ sets. This is particularly evident when comparing the YIS method with the 2000TT and the $24 \times 100 \mathrm{~m}$ set, where all the values were within $0.5 \mathrm{sec}$ of the combined mean LTV. The FSM values were consistently faster (up to $1.0 \mathrm{sec}$ ) and the $10 \times 300 \mathrm{~m}$ values consistently slower (again up to $1.0 \mathrm{sec}$ ) than the YIS method (see Table 2).

\section{Discussion}

The present study has outlined a method of assessing the LTV in highly trained swimmers. The YIS method calculates the lactate threshold as a function of the blood lactate - velocity curve, using the slope and the intercept. Where this is different to previous methods is that the slope is individual rather than fixed. Adding the intercept also allows a further individualization of the assessment that is not available from a single bestfit arrangement. Although the profiles of swimmers who took part in this study showed an exponential relationship between velocity and blood lactate, there were individual differences in slope angles that would have been missed using previous methods. The findings of studies using both an escalation test [1] and a discontinuous ramp test examining the relationship between exercise intensity and blood lactate concentration [13] can be more closely and accurately determined by using the YIS method. These data also demonstrate the validity of the YIS method to predict an appropriate pace for swimmers in shorter repetition distances.

A number of researchers have suggested that a blood concentration of $4 \mathrm{mM}$ can be used as the criterion value for identifying the $L T[2,3,6]$. Most of the studies on the determination of the LT and LTV have been conducted with running or cycling. These are generally longer activities that involve different muscle recruitment patterns that could affect LTV value $[3,19]$. Endurance-trained athletes may have a lower individual lactate concentration at LT than other athletes [1]. The mean lactate concentration at the LT for freestyle found by the YIS method ( $3.2 \mathrm{mM}$ ) was higher than that suggested in previous papers (3.0 $\mathrm{mM})[20]$ as a set value that could be assumed by using an FSM method and was significantly lower than that found by the FSM method $(3.4 \mathrm{mM})$ in this study.

The LTV is suggested to be representative of the point where purely aerobic work becomes partially anaerobic $[11,21]$. The amount of anaerobic work being performed at this point is not readily identifiable with routine lactate testing. The potential for overestimating the anaerobic contribution when using shorter duration intervals in a ramp test is greater than with a continuous ramp method, where there is less potential for working 
higher than the LTV speed. This assertion is supported by observations that discontinuous methods can over-estimate work intensities at the LTV [6].

Results obtained from discontinuous methods must be carefully interpreted to ensure that the correct intensity is achieved. The YIS method offers the ability to identify the specific LTv for each individual swimmer. Rather than if the LT occurs at a specific lactate concentration above the designated aerobic threshold (i.e. aerobic threshold $(\mathrm{mM})+1.0 \mathrm{mM}$, or at a set lactate of 3 $\mathrm{mM}$ ) [20], or a fixed lactate concentration (e.g. $4 \mathrm{mM}$ ) [21]. Individualization of testing elite athletes is a legitimate conceptual approach and the YIS method allows the individual LTv to be calculated for each profile.

Where pace was considered, no significant difference was found between the estimation of swim speed, as determined by the two methods. Although the difference between the two methods was not significantly different, in practical training terms, a 1 second difference in repeat times can make the difference between successfully completing a training set at a set pace over 2000-3000 m and seeing the swimmer unable to maintain pace, stroke characteristics, and therefore performance outcome. There were at least 10 subjects where the variation in pace determined by the YIS method and FSM method was over 3 seconds and up to 8 seconds in disagreement. This would cause a likelihood of training failure if these pace times were to be set at this level. Over the six-week study period, this model elicited a high correlation $(r=0.98)$ between the identification of the relative " $Y$ " axis occurrence of the LTV while still showing left/right changes in the lactate profile against speed. The high correlation between YIS and the practical assessments demonstrated the stability and predictive nature of the YIS method. Most of the training classification systems used in competitive sports are based on the accurate determination of the LTV. Although the YIS model used the $7 \times 200 \mathrm{~m}$ step test, it appears to be robust and transferable to a range of monitoring scenarios.

In conclusion, the YIS method for determining the LTV of swimmers is based on the y-intercept and slope of the lactate profile and is sufficiently sensitive to identify training-induced changes over a period of six-weeks. The formula LTV $=-0.217 \times[y$-intercept $($ La-V $)) /($ slope (La-V)] provides an objective method for evaluating the lactate-velocity curves in trained swimmers. Test retest relationships are strong and represent a high level of reproducibility. The validation methods support that the newly proposed YIS method is an accurate and valid means of determining the LT and LTV in highly-trained swimmers. This method is more likely to produce an effective estimation of the correct workload for the intensity based upon the LTV in short duration repetitions. In this respect, when testing elite swimmers, the YIS method represents a step forward from the existing methods, of which the FSM [6] model appeared to be the best. Caution is suggested in applying the model to different groups until further studies have been carried out.

\section{Acknowledgements}

The authors gratefully acknowledge the assistance and cooperation of the Australian and New Zealand swimmers in study and particularly the swimming coaches (Mark Regan, Gennadi Touretski, Barry Prime and Jim Fowlie) at the Australian Institute of Sport. Excellent technical assistance was provided by staff from the Department of Physiology, Australian Institute of Sport.

\section{References}

1. Borch KW, Ingjer F, Larsen S, Tomten SE (1993) Rate of accumulation of blood lactate during graded exercise as a predictor of 'anaerobic threshold'. J Sports Sci 11: 49-55.

2. Mader A, Heck H, Hollman W (1976) Evaluation of lactic acid anaerobic energy contribution by determination of post exercise lactic acid concentration of ear capillary blood in middle distance runners and swimmers. The International Congress of Physical Activity SCiences. Quebec 4: 187199.

3. Reilly T (1990) Swimming. In: Reilly T, Secher N, Snell P, Physiology of Sports. Melbourne, Chapman and Hall, 217257.

4. Yeh MP, Gardner RM, Adams TD, Yanowitz FG, Crapo RO (1983) "Anaerobic threshold": problems of determination and validation. J Appl Physiol Respir Environ Exerc Physiol 55: 1178-1186.

5. Billat LV (1996) Use of blood lactate measurements for prediction of exercise performance and for control of training. Recommendations for long-distance running. Sports Med 22: 157-175.

6. Heck H, Mader A, Hess G, Mücke S, Müller R, et al. (1985) Justification of the 4-mmol/l lactate threshold. Int J Sports Med 6: 117-130.

7. Sjodin B, Jacobs I (1981) Onset of blood lactate accumulation and marathon running performance. Int J Sports Med 2: 23-26.

8. Anderson GS, Rhodes EC (1989) A review of blood lactate and ventilatory methods of detecting transition thresholds. Sports Med 8: 43-55.

9. Bishop D, Jenkins DG, Mackinnon LT (1998) The relationship between plasma lactate parameters, Wpeak and 1-h cycling performance in women. Med Sci Sports Exerc 30: 1270-1275.

10. Bishop P, Martino M (1993) Blood lactate measurement in recovery as an adjunct to training. Practical considerations. Sports Med 16: 5-13.

11. Harrison JR, Dawson BT, Lawrence S, Blanksby BA (1992) Non-invasive and invasive determinations of the individual anaerobic thresholdin competitive swimmers. J Swim Res 8: 11-17.

12. Astrand PO, Rodahl K (1986) Textbook of work Physiology. ( $3^{\text {rd }}$ edn), The physiological basis of exercise, 304

13. Hughson RL, Weisiger KH, Swanson GD (1987) Blood lactate concentration increases as a continuous function in progressive exercise. J Appl Physiol (1985) 62: 1975-1981. 
14. Jones NL, Ehrsam RE (1982) Anaerobic threshold. In: Holloszy JO, Exercise and Sport Sciences Reviews. William and Wilkins, Baltimore, 49-83.

15. Simon G, Goerttler I, Kübel R, Kindermann W, Dickhuth $\mathrm{HH}$, et al. (1979) [Performance of cross-country skiers over a year's period]. Schweiz Z Sportmed 27: 5-19.

16. Pyne D, Maw G, Goldsmith W (2000) Physiological testing of elite swimmers. In: Gore CJ, Physiological Tests for Elite Athletes. Human Kinetics, Champaign, Illinois, 354-368.

17. Pyne DB, Lee H, Swanwick KM (2001) Monitoring the lactate threshold in world-ranked swimmers. Med Sci Sports Exerc 33: 291-297.
18. Cheng B, Kuipers $H$, Snyder AC, Keizer HA, Jeukendrup A, et al. (1992) A new approach for the determination of ventilatory and lactate thresholds. Int J Sports Med 13: 518-522.

19. Hellemans J (2000) The training intensity handbook for endurance sport. KinEli publishing.

20. Physiology of Sport and Exercise (1994) Champaign, IL: Human Kinetics, JH Wilmore and DL Costill, 109.

21. Stegmann H, Kindermann W (1982) Comparison of prolonged exercise tests at the individual anaerobic threshold and the fixed anaerobic threshold of $4 \mathrm{mmol} . \mathrm{I}(-1)$ lactate. Int J Sports Med 3: 105-110. 